\title{
Steiner systems and configurations of points
}

\author{
Edoardo Ballico $^{1} \cdot$ Giuseppe Favacchio $^{2} \cdot$ Elena Guardo $^{2}$ (D) $\cdot$ Lorenzo Milazzo $^{2}$
}

Received: 17 September 2019 / Revised: 29 June 2020 / Accepted: 13 October 2020 /

Published online: 31 October 2020

(c) The Author(s) 2020

\begin{abstract}
The aim of this paper is to make a connection between design theory and algebraic geometry/commutative algebra. In particular, given any Steiner System $S(t, n, v)$ we associate two ideals, in a suitable polynomial ring, defining a Steiner configuration of points and its Complement. We focus on the latter, studying its homological invariants, such as Hilbert Function and Betti numbers. We also study symbolic and regular powers associated to the ideal defining a Complement of a Steiner configuration of points, finding its Waldschmidt constant, regularity, bounds on its resurgence and asymptotic resurgence. We also compute the parameters of linear codes associated to any Steiner configuration of points and its Complement.
\end{abstract}

Keywords Steiner systems · Monomial ideals - Symbolic powers - Stanley Reisner rings · Linear codes

Mathematics Subject Classification 51E10 $\cdot 13 \mathrm{~F} 55 \cdot 13 \mathrm{~F} 20 \cdot 14 \mathrm{G} 50 \cdot 94 \mathrm{~B} 27$

\section{Introduction}

Combinatorial design theory is the study of arranging elements of a finite set into patterns (subsets, words, arrays) according to specified rules. It is a field of combinatorics connected to several other areas of mathematics including number theory and finite geometries. In the

Communicated by G. Korchmaros.

$凶 \quad$ Elena Guardo

guardo@dmi.unict.it

http://www.dmi.unict.it/guardo

Edoardo Ballico

edoardo.ballico@unitn.it

Giuseppe Favacchio

favacchio@dmi.unict.it

https://sites.google.com/view/giuseppefavacchio

Lorenzo Milazzo

milazzo@dmi.unict.it

1 Dipartimento di Matematica, Via Sommarive, 14, 38123 Povo, TN, Italy

2 Dipartimento di Matematica e Informatica, Viale A. Doria, 6, 95100 Catania, Italy 
last years, the main techniques used in combinatorial and algebraic geometry allow design theory to grow up involving applications in other areas such as in coding theory, cryptography, and computer science.

A $t-(v, n, \lambda)$-design $D=(V, B)$ is a pair consisting of a set $V$ of $v$ points and a collection $B$ of $n$-subsets of $V$, called blocks, such that every $t$-subset (or $t$-tuple) of $V$ is contained in exactly $\lambda$ blocks in $B$.

The numbers $v=|V|, b=|B|, n, \lambda$, and $t$ are called the parameters of the design.

A Steiner system $(V, B)$ of type $S(t, n, v)$ is a $t-(v, n, 1)$ design, that is, a collection $B$ of $n$-subsets (blocks) of a $v$-set $V$ such that each $t$-tuple of $V$ is contained in a unique block in $B$. The elements in $V$ are called vertices or points and those of $B$ are called blocks. In particular, a Steiner triple system of order $v, S T S(v)$, is a collection of triples (3-subsets) of $V$, such that each unordered pair of elements is contained in precisely one block, and a Steiner quadruple system of order $v, S Q S(v)$, is a collection of quadruples (4-subsets) of $V$ such that each triple is found in precisely one block.

A geometric study of some particular classes of Steiner systems, with an eye on the automorphism groups, can be found in [57]. There exists a very extensive literature on STSs and SQS, e.g. [5,6,11-13,27-30,37,40-45,47] to cite some of them.

In this paper we study special configurations of reduced points in $\mathbb{P}^{n}$ constructed on Steiner Systems, combining Combinatorial Algebraic Geometry and Commutative Algebra. We found many results concerning geometric and algebraic codes and Steiner systems (or designs in general) but we did not find in the literature any result concerning the study of combinatorial structures, as Steiner systems, using their homological invariants. Thus, we consider this paper as a starting point of the study of combinatorial designs, such as Steiner systems, using the homological invariants of their defining ideals. An introduction of coding theory with the use of Algebraic Geometry can be found in [56]. In particular, we can refer to [48] for some techniques used to study algebraic varieties with special combinatorial features. Recently, connections between commutative algebra and coding theory have gained much attention (see [15,16,19,24,31,34,38,52-54] for more results in this direction). For a background in commutative algebra with a view toward algebraic geometry we suggest [20].

The paper is structured as follow. Section 2 provides the background on designs, ideals of points, symbolic and regular powers of ideals, containment problem and monomial ideals. We introduce two finite sets of reduced points in $\mathbb{P}^{n}$ called Steiner configuration and Complement of a Steiner configuration (see Definitions 2.3 and 2.4). As pointed out in Remark 2.5, a Steiner configuration of points and its Complement constructed from a Steiner system of type $S(t, n, v)$ are subschemes of a so called star configuration of $\left(\begin{array}{l}v \\ n\end{array}\right)$ points in $\mathbb{P}^{n}$.

In Sect. 3, we will focus on the Complement of a Steiner configuration of points because it is a proper hyperplanes section of a monomial ideal that is the Stanley-Reisner ideal of a matroid (see Theorem 3.4). Thus, each $m$-th symbolic power of such a monomial ideal is arithmetically Cohen-Macaulay and, after a proper hyperplanes section, it agrees with the $m$-th symbolic power of the ideal defining a Complement of a Steiner configuration (see Proposition 3.6). This connection allows us to study the homological invariants of the ideal $I_{X_{C}}$ of the Complement of a Steiner configuration. We start with the description of the initial degree $I_{X_{C}}$, i.e. we describe the minimum integer $d$ such that $\left(I_{X_{C}}\right)_{d} \neq(0)$ or, equivalently, the least degree $d$ of a minimal generator of $I_{X_{C}}$. We also compute its Waldschmidt constant and give results on the containment problem of $I_{X_{C}}$, i.e. the problem of determining for which $m$ and $d$ the containment $I_{X_{C}}^{(m)} \subseteq I_{X_{C}}^{d}$ holds. Section 4 is devoted to the computation of the Hilbert Function, the regularity and graded Betti numbers of $I_{X_{C}}$. In particular, we give some bounds for the asymptotic resurgence and the resurgence of $I_{X_{C}}$. 
Unfortunately, the monomial ideal associated to a Steiner configuration of points does not lead to a Cohen-Macaulay algebra and we cannot apply the same methods as for its Complement. From a combinatorial point of view, two Steiner systems having the same parameters could have very different properties. Computational experiments give us examples where such differences effect the homological invariants. We pose Questions 4.15 and 4.16 on the behaviour of Steiner configurations of points to lead us and the interested researcher towards future works.

We end the paper with an application to coding theory. Following [15,24,52-55], we study special linear codes associated to a Steiner configuration of points and its Complement. In particular, their combinatorial structure allows us to compute the Hamming distance of the associated code (Proposition 5.2 and Theorem 5.4).

The computer softwares CoCoA [1] and Macaulay2 [32] were indispensable for all the computations we made.

\section{Preliminaries and notation}

Steiner systems play an important role in Design Theory. We use [11] and [13] as main references for all the background on this topic.

The existence of a Steiner system strongly depends on the parameters $(t, n, v)$. For instance if $t=2$ and $n=3$ then $v \equiv 1,3 \bmod (6)$ must hold. There are known necessary conditions for the existence of a Steiner system of type $S(t, n, v)$ that are not in general sufficient. If a Steiner system $(V, B)$ of type $S(t, n, v)$ exists, then $|B|=\frac{\left(\begin{array}{c}v \\ t\end{array}\right)}{\left(\begin{array}{c}n \\ t\end{array}\right)}$.

Example 2.1 One of the simplest and most known examples of Steiner system is the Fano Plane. It is unique up to isomorphism and it is a Steiner system of type $S(2,3,7)$ with block set

$$
B:=\{\{1,2,3\},\{1,4,5\},\{1,6,7\},\{2,4,6\},\{2,5,7\},\{3,4,7\},\{3,5,6\}\} .
$$

\subsection{Ideals of Steiner configuration of points and its complement}

Let $V$ be a set of $v$ points and $\mathcal{H}:=\left\{H_{1}, \ldots H_{v}\right\}$ be a collection of distinct hyperplanes $H_{j}$ of $\mathbb{P}^{n}$ defined by the linear forms $\ell_{j}$ for $j=1 \ldots, n$ and $n \leq v$. Assume that any $n$ hyperplanes in $\mathcal{H}$ meet in a point. There is a natural way to associate a point in $\mathbb{P}^{n}$ to a $n$-subset of $V$. Indeed, given a $n$-subset $\sigma:=\left\{\sigma_{1}, \ldots \sigma_{n}\right\}$ of $V$, we denote by $P_{\mathcal{H}, \sigma}$ the point intersection of the hyperplanes $H_{\sigma_{1}}, \ldots, H_{\sigma_{n}}$. Then the ideal $I_{P_{\mathcal{H}, \sigma}}=\left(\ell_{\sigma_{1}}, \ldots, \ell_{\sigma_{n}}\right) \subseteq k\left[\mathbb{P}^{n}\right]$ is the vanishing ideal of the point $P_{\mathcal{H}, \sigma}$.

Definition 2.2 Given a set $V$ of $v$ points and a general collection $Y$ of subsets of $V$, we define the following set of points in $\mathbb{P}^{n}$ with respect to $\mathcal{H}$

$$
X_{\mathcal{H}, Y}:=\cup_{\sigma \in Y} P_{\mathcal{H}, \sigma}
$$

and its defining ideal

$$
I_{X_{\mathcal{H}, Y}}:=\cap_{\sigma \in Y} I_{P_{\mathcal{H}, \sigma}} .
$$

In particular, if $n \leq v$ are positive integers and $V$ is a set of $v$ points, we denote by $C_{(n, v)}$ the set containing all the $n$-subsets of $V$. 
Definition 2.3 Let $(V, B)$ be a Steiner system of type $S(t, n, v)$ with $t<v \leq n$. We associate to $B$ the following set of points in $\mathbb{P}^{n}$

$$
X_{\mathcal{H}, B}:=\cup_{\sigma \in B} P_{\mathcal{H}, \sigma}
$$

and its defining ideal

$$
I_{X_{\mathcal{H}, B}}:=\cap_{\sigma \in B} I_{P_{\mathcal{H}, \sigma}} .
$$

We call $X_{\mathcal{H}, B}$ the Steiner configuration of points associated to the Steiner system $(V, B)$ of type $S(t, n, v)$ with respect to $\mathcal{H}$ (or just $X_{B}$ if there is no ambiguity).

Definition 2.4 Let $(V, B)$ be a Steiner system of type $S(t, n, v)$ with $t<n \leq v$. We associate to $C_{(n, v)} \backslash B$ the following set of points in $\mathbb{P}^{n}$

$$
X_{\mathcal{H}, C_{(n, v)} \backslash B}:=\cup_{\sigma \in C_{(n, v)} \backslash B} P_{\mathcal{H}, \sigma}
$$

and its defining ideal

$$
I_{X_{\mathcal{H}, C_{(n, v)} \backslash B}}:=\bigcap_{\sigma \in C_{(n, v)} \backslash B} I_{P_{\mathcal{H}, \sigma}} .
$$

We call $X_{\mathcal{H}, C_{(v, n)} \backslash B}$ the Complement of a Steiner configuration of points with respect to $\mathcal{H}$ (or C-Steiner $X_{\mathrm{C}}$ if there is no ambiguity).

Remark 2.5 Note that from Definition 2.2, it follows that $X_{\mathcal{H}, C_{(n, v)}}$ is a so called star configuration of $\left(\begin{array}{l}v \\ n\end{array}\right)$ points in $\mathbb{P}^{n}$. In particular, from Definitions 2.3 and $2.4, X_{\mathcal{H}, C_{(n, v)} \backslash B}$ is the Complement of the Steiner configuration $X_{\mathcal{H}, B}$ in the star configuration $X_{\mathcal{H}, C_{(n, v)}}$. Thus, a Steiner configuration of points and its Complement are subschemes of a star configuration of $\left(\begin{array}{l}v \\ n\end{array}\right)$ points in $\mathbb{P}^{n}$. See for instance [7-9,25,26] for recent results on star configurations and see [55] for the connections between linear codes and ideals of star configurations.

Remark 2.6 Since the set $X_{\mathcal{H}, B}$ contains $|B|$ points, we have that

$$
\operatorname{deg} X_{\mathcal{H}, C_{(n, v)} \backslash B}=\left(\begin{array}{l}
v \\
n
\end{array}\right)-|B|=\left(\begin{array}{l}
v \\
n
\end{array}\right)-\frac{\left(\begin{array}{l}
v \\
t
\end{array}\right)}{\left(\begin{array}{l}
n \\
t
\end{array}\right)} .
$$

Example 2.7 Consider the Steiner configuration associated to $(V, B)$ of type $S(2,3,7)$ as in Example 2.1. Take $\mathcal{H}:=\left\{H_{1}, \ldots, H_{7}\right\}$ a collection of 7 distinct hyperplanes $H_{i}$ in $\mathbb{P}^{3}$ defined by a linear form $\ell_{i}$ for $i=1, \ldots, 7$, respectively, with the property that any 3 of them meet in a point $P_{\mathcal{H}, \sigma}=H_{\sigma_{1}} \cap H_{\sigma_{2}} \cap H_{\sigma_{3}}$, where $\sigma=\left\{\sigma_{1}, \sigma_{2}, \sigma_{3}\right\} \in B$. We get that $X_{\mathcal{H}, C_{(3,7)}}$ is a star configuration of $\left(\begin{array}{l}7 \\ 3\end{array}\right)=35$ points in $\mathbb{P}^{3}, X_{\mathcal{H}, B}:=\cup_{\sigma \in B}\left\{P_{\mathcal{H}, \sigma}\right\}$ is a Steiner configuration consisting of 7 points in $\mathbb{P}^{3}$ and $X_{\mathcal{H}, C_{(3,7)} \backslash B}$ is a C-Steiner configuration consisting of $\left(\begin{array}{l}7 \\ 3\end{array}\right)-7=28$ points in $\mathbb{P}^{3}$. Their defining ideals are respectively,

$$
I_{X_{\mathcal{H}, B}}:=\cap_{\sigma \in B} I_{P_{\mathcal{H}, \sigma}} \text { and } I_{X_{\mathcal{H}, C_{(3,7)} \backslash B}}:=\bigcap_{\sigma \in C_{(3,7)} \backslash B} I_{P_{\mathcal{H}, \sigma}} .
$$

\subsection{Symbolic and regular powers of an ideal and the containment problem}

In this section, we recall some definitions and known results concerning the symbolic and regular powers of ideal of points. 
Let $I$ be a homogeneous ideal in the standard graded polynomial ring $R:=k\left[x_{0}, \ldots, x_{n}\right]$. Given an integer $m$, we denote by $I^{m}$ the regular power of the ideal $I$. The $m$-th symbolic power of $I$ is defined as

$$
I^{(m)}=\bigcap_{\mathfrak{p} \in \operatorname{Ass}(I)}\left(I^{m} R_{\mathfrak{p}} \cap R\right)
$$

where $\operatorname{Ass}(I)$ denotes the set of associated primes of $I$. If $I$ is a radical ideal (this includes for instance squarefree monomial ideals and ideals of finite sets of points) then

$$
I^{(m)}=\bigcap_{\mathfrak{p} \in A s s(I)} \mathfrak{p}^{m} .
$$

It always holds that $I^{m} \subseteq I^{(m)}$. In particular, the containment problem is of interest, i.e. the problem of determining for which $m$ and $d$ the containment $I^{(m)} \subseteq I^{d}$ holds. We refer the reader to $[2-4,17,18,36,50]$ for a partial list of papers on this topic.

The investigation of this problem led to the introduction of other invariants with the aim of comparison between symbolic and ordinary powers of an ideal.

Let $I$ be a homogeneous ideal, the real number

$$
\rho(I)=\sup \left\{\frac{m}{r}: I^{(m)} \nsubseteq I^{r}\right\}
$$

is called resurgence of $I$ (see [3,4]). For a homogeneous ideal (0) $\subset I \subset k\left[\mathbb{P}^{n}\right]$, in [33] the authors define an asymptotic resurgence as follows:

$$
\rho_{a}(I)=\sup \left\{\frac{m}{r}: I^{(m t)} \nsubseteq I^{r t} \text { for all } t>>0\right\} .
$$

The resurgence is strictly related to the Waldschmidt constant of $I$, introduced in [59] in a completely different setting. If $\alpha(I)$ is the minimum integer $d$ such that $I_{d} \neq(0)$, then the Waldschmidt constant of $I, \widehat{\alpha}(I)$, is the real number

$$
\widehat{\alpha}(I)=\lim _{m \rightarrow \infty} \frac{\alpha\left(I^{(m)}\right)}{m} .
$$

Given $r$ distinct points $P_{i} \in \mathbb{P}^{n}$ and non-negative integers $m_{i}$, we denote by $Z=m_{1} P_{1}+$ $\cdots+m_{r} P_{r} \subset \mathbb{P}^{n}$ the set of fat points defined by $I_{Z}=\bigcap_{i} I_{P_{i}}^{m_{i}}$, where $I_{P_{i}}$ is the prime ideal generated by all forms which vanish at $P_{i}$. The set $X=\left\{P_{1}, \ldots, P_{r}\right\}$ is called the support of $Z$. When $m_{i}=m$ for all $i, I_{Z}=\bigcap_{i} I_{P_{i}}^{m}=I_{X}^{(m)}$.

If $I$ is the ideal of a set of (fat) points, we denote by $I_{i}$ (resp. $R_{i}$ ) the vector space span in $I$ (resp. $R$ ) of the forms of degree $i$ in $I$ (resp. $R$ ). The function $H: \mathbb{N} \rightarrow \mathbb{N}$ such that $H(i)=\operatorname{dim}_{k}(R / I)_{i}=\operatorname{dim}_{k} R_{i}-\operatorname{dim}_{k} I_{i}$ is called the Hilbert Function of $R / I$ and it is denoted by $H_{R / I}(i)$. The Castelnuovo-Mumford regularity of $I$, denoted by $\operatorname{reg}(I)$, is the least degree $t>0$ such that $\operatorname{dim}(R / I)_{t}=\operatorname{dim}(R / I)_{t-1}$.

Theorem 1.2.1 in [4] shows that if $I$ defines a 0 -dimensional scheme, i.e., a finite set of points, then

$$
\frac{\alpha(I)}{\widehat{\alpha}(I)} \leq \rho(I) \leq \frac{\operatorname{reg}(I)}{\widehat{\alpha}(I)}
$$

In [33], Theorem 1.2. shows that (1) $1 \leq \frac{\alpha(I)}{\widehat{\alpha}(I)} \leq \rho_{a}(I) \leq \rho(I) \leq h$ where $h=$ $\min \left(N, h_{I}\right)$ and $h_{I}$ is the maximum of the heights of the associated primes of $I$; (2) if $I$ is the ideal of a (non-empty) smooth subscheme of $\mathbb{P}^{n}$, then $\rho_{a}(I) \leq \frac{\omega(I)}{\widehat{\alpha}(I)} \leq \frac{\operatorname{reg}(I)}{\widehat{\alpha}(I)}$ where $\omega(I)$ is the largest degree in a minimal homogeneous set of generators of $I$. 


\subsection{Monomial ideals, simplicial complexes}

In this section, we show how monomial ideals form an important link between commutative algebra and combinatorics. A monomial ideal is uniquely determined by the monomials it contains. Monomial ideals also arise in graph theory. Given a graph $G$ with vertices $\left\{x_{1}, \ldots, x_{v}\right\}$, we associate the ideal $I_{G}$ in $k\left[x_{1}, \ldots, x_{v}\right]$ generated by the quadratic monomials $x_{i} x_{j}$ such that $x_{i}$ is adjacent to $x_{j}$. From known results in the literature, it is possible to determine many invariants for monomial ideals.

In particular, ideals generated by squarefree monomials have a beautiful combinatorial interpretation in terms of simplicial complexes. In the next sections, we will use these properties to completely describe the most important homological invariants of an ideal defining a Complement of a Steiner configuration of points. This is equivalent to say that we are describing special subsets of star configurations using tools from combinatorics and algebraic geometry. Unfortunately, the ideal defining a Steiner configuration of points cannot be described using monomial ideals, and we cannot determine its homological invariants. So, the problem is still open. At the end of the paper, we will be able to describe the parameters of the linear codes associated to both a Steiner configuration of points and its Complement.

We refer to [35] for notation and basic facts on monomial ideals and to [4] for an extensive coverage of the theory of Stanley-Reisner ideals.

Definition 2.8 An ideal $I$ in a polynomial ring $R$ is called a monomial ideal if there is a subset $\mathcal{A} \subset \mathbb{Z}_{\geq 0}^{n}$ (possible infinite) such that $I$ consists of all polynomials of the form $\sum_{\alpha \in \mathcal{A}} h_{\alpha} x^{\alpha}$, where $\bar{h}_{\alpha} \in R$.

Definition 2.9 A simplicial complex $\Delta$ over a set of vertices $V=\left\{x_{1}, \ldots, x_{v}\right\}$ is a collection of subsets of $V$ satisfying the following two conditions:

(1) $\left\{x_{i}\right\} \in \Delta$ for all $1 \leq i \leq v$

(2) if $F \in \Delta$ and $G \subset F$, then $G \in \Delta$.

An element $F$ of $\Delta$ is called a face, and the dimension of a face $F$ of $\Delta$ is $|F|-1$, where $|F|$ is the number of vertices of $F$. The faces of dimensions 0 and 1 are called vertices and edges, respectively, and $\operatorname{dim} \emptyset=-1$.

The maximal faces of $\Delta$ under inclusion are called facets of $\Delta$. The dimension of the simplicial complex $\Delta$ is $\operatorname{dim} \Delta=\max \{\operatorname{dim} F \mid F \in \Delta\}$. We refer to $i$-dimensional faces as $i$-faces. We denote the simplicial complex $\Delta$ with facets $F_{1}, \ldots, F_{q}$ by $\Delta=\left\langle F_{1}, \ldots, F_{q}\right\rangle$ and we call $\left\{F_{1}, \ldots, F_{q}\right\}$ the facet set of $\Delta$.

If $\Delta$ is a $d$-dimensional simplicial complex, the most important invariant is the $f$-vector (or face vector) of $\Delta$ and it is denoted by $\left(f_{0}, \ldots, f_{d}\right) \in \mathbb{N}^{d+1}$, where $f_{i}$ denotes the number of $i$-dimensional faces in $\Delta$. From the monomial ideal point of view, the $f$-vector is encoded in the Hilbert series of the quotient ring $R / I_{\Delta}$ and it is related to the $h$-vector of a suitable set of points in $\mathbb{P}^{n}$ (see Sect. 4). We will use Remark 2.11 to show the connections between Stanley-Reisner ideals, simplicial complexes, matroids and Steiner systems to determine Waldschmidt constant and bounds for the resurgence (see Sect. 3), Betti numbers, Hilbert function, and regularity of the ideal of a Complement of a Steiner configurations of points (see Sect. 4).

The Alexander dual of a simplicial complex $\Delta$ on $V=\left\{x_{1}, \ldots, x_{v}\right\}$ is the simplicial complex $\Delta^{\vee}$ on $V$ with faces $V \backslash \sigma$, where $\sigma \notin \Delta$.

The Stanley-Reisner ideal of $\Delta$ is the ideal $I_{\Delta}:=\left(x^{\sigma} \mid \sigma \notin \Delta\right)$ of $R=k\left[x_{1}, \ldots, x_{v}\right]$, where $x^{\sigma}=\Pi_{i \in \sigma} x_{i}$. It is well known that the Stanley-Reisner ideals are precisely the 
squarefree monomial ideals. The quotient ring $k[\Delta]:=R / I_{\Delta}$ is the Stanley-Reisner ring of the simplicial complex $\Delta$.

Each simplicial complex has a geometric realization as a certain subset of a finite dimensional affine space.

If $V$ is a set $v$ points, we denote by $k[V]:=k\left[x_{1}, \ldots, x_{v}\right]$ the standard graded polynomial ring in $v$ variables. Given a $n$-subset of $V, \sigma:=\left\{i_{1}, i_{2}, \ldots, i_{n}\right\} \subseteq V$, we will write

$$
\mathfrak{p}_{\sigma}:=\left(x_{i_{1}}, x_{i_{2}}, \ldots, x_{i_{n}}\right) \subseteq k[V]
$$

for the prime ideal generated by the variables indexed by $\sigma$, and

$$
M_{\sigma}:=x_{i_{1}} x_{i_{2}} \cdots x_{i_{n}} \in k[V]
$$

for the monomial given by the product of the variables indexed by $\sigma$.

Let $n \leq v$ be positive integers, and $V$ a set of $v$ points; recall that $C_{(n, v)}$ is the set containing all the $n$-subsets of $V$.

Definition 2.10 If $T \subset C_{(n, v)}$, we define two ideals

$$
I_{T}:=\left(M_{\sigma} \mid \sigma \in T\right) \subseteq k[V]
$$

and

$$
J_{T}:=\bigcap_{\sigma \in T} \mathfrak{p}_{\sigma} \subseteq k[V]
$$

called the face ideal of $T$ and the cover ideal of $T$, respectively.

Remark 2.11 It is well known that $J_{T}$ is the Stanley-Reisner ideal $I_{\Delta_{T}}$ of the simplicial complex

$$
\Delta_{T}:=\langle V \backslash \sigma \mid \sigma \in T\rangle .
$$

Then $J_{T}$ is generated by the monomials $M_{b}$ with $b \notin \Delta_{T}$. We also recall that $I_{T}$ and $J_{T}$ are the Alexander duals of each other.

Since $J_{T}$ is a squarefree monomial ideal, the $m$-th symbolic power of $J_{T}$ (Theorem 3.7 in $[14])$ is

$$
J_{T}^{(m)}:=\bigcap_{\sigma \in T} \mathfrak{p}_{\sigma}^{m}
$$

\section{Matroid and configurations of points from Steiner systems: Waldschmidt constant and containment problem}

This section is devoted to show how the results of the previuos sections are related to our special configurations of points. In particular, we will show that the Complement of a Steiner configuration of points is connected with the theory of matroids.

Definition 3.1 A simplicial complex $\Delta$ is said to be a matroid if $F, G \in \Delta$ and if $|F|>|G|$ then there exists $i \in F \backslash G$ such that $G \cup\{i\} \in \Delta$.

We also recall that we say that a homogeneous ideal $J$ in a polynomial ring $R$ is CohenMacaulay $(\mathrm{CM})$ if $R / J$ is Cohen-Macaulay, i.e. $\operatorname{depth}(R / J)=\operatorname{Krull}-\operatorname{dim}(R / J)$. Varbaro in [58] and Minh and Trung in [46] have independently shown the following 
Theorem 3.2 (Varbaro [58], Minh and Trung [46]) Let $\Delta$ be a simplicial complex. Then $k[V] / I_{\Delta}^{(m)}$ is Cohen-Macaulay for each $m \geq 1$ if and only if $\Delta$ is a matroid.

Terai and Trung in [51] proved if $I_{\Delta}^{(m)}$ is Cohen-Macaulay for some $m \geq 3$ then $\Delta$ is a matroid.

To shorten the notation, from now on we set $C:=C_{(n, v)} \backslash B$. Let $X_{\mathcal{H}, B}$ be a Steiner configuration of points and $X_{\mathcal{H}, C}$ its Complement as previous defined with respect to a collection $\mathcal{H}$ of hyperplanes in $\mathbb{P}^{n}$.

We claim that $I_{X_{\mathcal{H}, C}}^{(m)}$ and $J_{C}^{(m)}$ share the same homological invariants for any positive integer $m$. In particular, the key point of our argument is that $J_{C}$ is the Stanley-Reisner ideal of the simplicial complex $\Delta_{C}$, and that $\Delta_{C}$ is a matroid (see Theorem 3.4).

Several times in the literature simplicial complexes have been associated to Steiner systems. See for instance [12,49]. In Example 4.6 in [18] the well known Fano matroid is used to construct a Cohen-Macaulay ideal $I$ such that $I^{(3)} \neq I^{3}$ and $I^{(2)} \neq I^{2}$. The quoted example is one of the cases described in Corollary 3.10.

We need the following auxiliary lemma.

Lemma 3.3 Let $(V, B)$ be a Steiner system of type $S(t, n, v)$. If $C_{(v-n-1, v)}$ is the set containing all the $(v-n+1)$-subsets of $V$, then $C_{(v-n-1, v)} \subseteq \Delta_{C}$.

Proof Let $F$ be a $(v-n-1)$-set of $V$. In order to prove the lemma we need to find $G \in C$ such that $F \subseteq V \backslash G$, i.e., $G \subseteq V \backslash F$. First note that $|V \backslash F|=n+1$, so we can take two $n$-subsets $G_{1}, G_{2}$ of $V \backslash F$ sharing a $n-1$-subset. Since $n-1 \geq t$, from the definition of Steiner Systems at least one of them, say $G_{1}$, does not belong to $G$. Then $G_{1} \in C$ and $G \subseteq V \backslash F$.

The next results are useful to describe the Complement of a Steiner configuration of points using the combinatorial properties of matroids.

Theorem 3.4 Let $(V, B)$ be a Steiner system of type $S(t, n, v)$. Then $\Delta_{C}$ is a matroid.

Proof Let $F, G$ be two maximal elements in $\Delta_{C}$, i.e., $F, G$ belong to the facet set of $\Delta_{C}$. Assume by contradiction $(F \backslash\{i\}) \cup\{j\} \notin \Delta_{C}$ for each $i \in F$ and for each $j \in F$. Then all the sets $\sigma_{i, j}:=V \backslash((F \backslash\{i\}) \cup\{j\})$ belong to $B$. Since $n \geq 2$, at least two blocks of $B$ share a $(n-1)$-set. That is a contradiction because $n-1>t-1$ from Definition 2.3.

Proposition 3.5 Let $(V, B)$ be a Steiner system of type $S(t, n, v)$. Then $k[V] / J_{C}^{(m)}$ is CohenMacaulay for each $m \geq 1$.

Proof Since $J_{C}=I_{\Delta_{C}}$, it is a consequence of Theorem 3.2 and Theorem 3.4.

Now, given a Steiner system of type $S(t, n, v)$ we are able to describe some homological invariants of the ideal associated to a Complement of Steiner configuration of points. Unfortunately, we have no similar result as Proposition 3.5 that should hold for a Steiner configuration of points.

We now prove the main claim of this section.

Proposition 3.6 $I_{X_{\mathcal{H}, C}}^{(m)} \subseteq k\left[\mathbb{P}^{n}\right]$ and $I_{\Delta_{C}}^{(m)} \subseteq k[V]$ share the same homological invariants.

Proof It is an immediate consequence of Theorem 3.6. in [26]. Indeed, $k[V] / I_{\Delta_{C}}^{(m)}$ is CohenMacaulay, and any subset of at most $n$ linear forms in $\left\{\ell_{1}, \ldots, \ell_{v}\right\}$ is a $k\left[\mathbb{P}^{n}\right]$-regular sequence. 
The Cohen-Macaulay property of $k[V] / I_{\Delta_{C}}^{(m)}$ also allows us to look at $I_{X_{\mathcal{H}, C}}^{(m)}$ as a proper hyperplane section of $I_{\Delta_{C}}^{(m)}$ (see [57]). This construction is quite standard but is very useful to describe combinatorial properties for arithmetically Cohen-Macaulay varieties $X$, i.e, $\operatorname{depth}\left(R / I_{X}\right)=\operatorname{Krull}-\operatorname{dim}\left(R / I_{X}\right)$, not only in projective spaces, but also in multiprojective spaces, see for instance [21-23,26]. The common idea is to relate, when possible, ideals of arithmetically Cohen-Macaulay varieties to monomial ideals in order to study their invariants.

We start with the description of the initial degree $\alpha\left(J_{C}\right)$ of $J_{C}=I_{\Delta_{C}}$, i.e. we describe the minimum integer $d$ such that $\left(J_{C}\right)_{d} \neq(0)$ or, equivalently, the least degree $d$ of a minimal generator of $J_{C}$. For the ease of the reader, we prove the following result that shows that $\left\{\alpha\left(J_{C}^{m}\right)\right\}_{m}$ is a strictly increasing sequence.

Lemma 3.7 Let $\mathfrak{p} \subseteq k[V]$ be a squarefree monomial ideal and $M \in \mathfrak{p}^{m}$. Then $\frac{\partial M}{\partial x_{j}} \in \mathfrak{p}^{m-1}$ for any $j \in V$.

Proof Set $\mathfrak{p}=\mathfrak{p}_{\sigma}$ where $\sigma \subseteq V$ and let $x_{1}^{a_{1}} \ldots x_{v}^{a_{v}} \in \mathfrak{p}^{m}$. Then $\sum_{i \in \sigma} a_{i} \geq m$. If $a_{j}=0$ then trivially $0 \in \mathfrak{p}^{m-1}$. If $a_{j}>0$ then $\frac{\partial M}{\partial x_{j}}=a_{j} x_{1}^{a_{1}} \ldots x_{j}^{a_{j}-1} \ldots x_{v}^{a_{v}}$ and $\sum_{i \in \sigma} a_{i} \geq m-1$.

Proposition 3.8 Let $(V, B)$ be a Steiner system of type $S(t, n, v)$. Then

(i) $\alpha\left(J_{C}\right)=v-n$;

(ii) $\alpha\left(J_{C}^{(q)}\right)=v-n+q$ for $2 \leq q<n$;

(iii) $\alpha\left(J_{C}^{(m)}\right)=\alpha\left(J_{C}^{(q)}\right)+p v$, where $m=p n+q$ and $0 \leq q<n$.

Proof (i) We have $J_{C}=I_{\Delta_{C}}=\left(M_{\sigma} \mid \sigma \notin \Delta_{C}\right)$. From Lemma 3.3 we have that the elements not in $\Delta_{C}$ have cardinality at least $v-n$. On the other hand, for any $\beta \in B$ we have $V \backslash \beta \notin \Delta_{C}$.

(ii) First we show $\alpha\left(J_{C}^{(q)}\right) \leq v-n+q$. Let $\beta \in B$ and $\sigma \subseteq \beta$ with $|\sigma|=q$, then we claim $M_{(V \backslash \beta) \cup \sigma} \in \alpha\left(J_{C}^{(q)}\right)$. Assume by contradiction $M_{(V \backslash \beta) \cup \sigma} \notin \mathfrak{p}_{\tau}^{q}$ for some $\tau \in V$. Then $(V \backslash \beta) \cap \sigma \cap \tau=\varnothing$ but this is a contradiction since $v-n+q+n>n$. On the other hand, if $q>2$ the statement follows by Lemma 3.7, since $\alpha\left(J_{C}^{(q)}\right)>\alpha\left(J_{C}^{(q-1)}\right)=$ $v-n+q-1$. If $q=2$ we proceed by contradiction. Let $M \in J_{C}^{(2)}$ be a monomial such that $\operatorname{deg} M=v-n+1$. Then there are at least $n-1$ variables that do not divide $M$. Since $t \leq n-1$, there is at most one block in $B$ containing these variables. Therefore there are at least $v-n$ elements of $C$ containing them. Since $M \in J_{C}^{(2)}, M$ belongs to each of these ideals to the power of 2, i.e., there are $v-n$ variables $x_{j}$ such that $\left.x_{j}^{2}\right|_{M}$. So $\operatorname{deg} M \geq 2(v-n)>v-n+1$.

(iii) We show that $J_{C}^{(m)}: M_{V}=J_{C}^{(m-n)}$.

- If $F \in J_{C}^{(m-n)}$ then $M_{V} F \in J_{C}^{(n)} \cdot J_{C}^{(m-n)} \subseteq J_{C}^{(m)}$.

- If $F M_{V} \in J_{C}^{(m)}$ then, by Lemma 3.7, $F M_{V \backslash \sigma} \in J_{C}^{(m-n)}$ for any $\sigma \in T$. Thus $F M_{V \backslash \sigma} \in$ $\mathfrak{p}_{\sigma}^{(m-n)}$. This implies $F \in \mathfrak{p}_{\sigma}^{(m-n)}$.

Then $\alpha\left(J_{C}^{(m)}\right)=\alpha\left(J_{C}^{(m-n)}\right)+v=\cdots=\alpha\left(J_{C}^{(q)}\right)+p v$, where $m=p n+q$ and $0 \leq q<n$.

From Proposition 3.6 and Proposition 3.8, the initial degree of the ideal of a C-Steiner configuration of points only depends on the parameters $(t, n, v)$ of the Steiner system.

Using the previous results, we have the following theorem: 
Theorem 3.9 Let $(V, B)$ be a Steiner system of type $S(t, n, v)$. Then

(i) $\alpha\left(I_{X_{C}}\right)=v-n$;

(ii) $\alpha\left(I_{X_{C}}^{(q)}\right)=v-n+q$, for $2 \leq q<n$;

(iii) $\alpha\left(I_{X_{C}}^{(m)}\right)=\alpha\left(I_{X_{C}}^{(q)}\right)+p v$, where $m=p n+q$ and $0 \leq q<n$ and $\alpha\left(I_{X_{C}}^{(n)}\right)=$ $\alpha\left(I_{X_{C}}^{(0)}\right)+v=v$.

An important consequence of Theorem 3.9 is related to the containment problem.

Corollary 3.10 Let $(V, B)$ be a Steiner system of type $S(t, n, v)$. Then $I_{X_{C}}^{(m)} \nsubseteq I_{X_{C}}^{d}$ for any pair $(m, d)$ such that

$$
\begin{aligned}
& m \equiv 1 \quad \bmod n \text { and } \quad d>1+\frac{(m-1) v}{n(v-n)} \quad \text { or } \\
& m \neq \equiv 1 \quad \bmod n \text { and } d>1+\frac{m-n}{n}+\frac{m}{(v-n)}
\end{aligned}
$$

In particular, if $v>2 n$ then

$$
I_{X_{C}}^{(n)} \nsubseteq I_{X_{C}}^{2}
$$

Proof From item $i)$ in Theorem $3.9 \alpha\left(I_{X_{C}}^{d}\right)=d(v-n)$. Therefore, it is enough to take $m$ such that $\alpha\left(I_{X_{C}}^{(m)}\right)<d(v-n)$. If $m \equiv 1 \bmod n$ then, from items $\left.i\right)$ and $\left.i i i\right)$ in Theorem 3.9, we get $\alpha\left(I_{X_{C}}^{(m)}\right)=(v-n)+\frac{(m-1) v}{n}$. Then $(v-n)+\frac{(m-1) v}{n}<d(v-n)$ implies the statement. If $m \neq \equiv 1 \bmod n$ then $m=p n+q$ and $2 \leq q \leq n$ and, from items $i i)$ and iii) in Theorem 3.9, we get $\alpha\left(I_{X_{C}}^{(m)}\right)=v-n+m-p n+p v=(1+p)(v-n)+m$. Thus $(1+p)(v-n)<d(v-n)+m$ implies $d>1+p+\frac{m}{(v-n)} \geq 1+\frac{m-n}{n}+\frac{m}{(v-n)}$.

In the case $m=n$, we get $1+\frac{m-n}{n}+\frac{m}{(v-n)}=1+\frac{n}{(v-n)}<2$.

Example 3.11 If $X:=X_{\mathcal{H}, C_{(3,7)} \backslash B} \subseteq \mathbb{P}^{3}$ is the C-Steiner configuration of points as in Example 2.7, then $I_{X}^{(3)} \nsubseteq I_{X}^{2}$.

Another immediate corollary allows us to compute the Waldschmidt constant of a CSteiner configuration. The Waldschmidt constant of a uniform matroid was computed in Theorem 7.5 in [2]. Properties of a uniform matroid were also studied in [26].

Corollary 3.12 If $(V, B)$ is a Steiner system of type $S(t, n, v)$, then the Waldschmidt constant of $I_{X_{C}}$ is

$$
\widehat{\alpha}\left(I_{X_{C}}\right)=\frac{v}{n}
$$

Proof From [10], Lemma 1, the limit $\widehat{\alpha}\left(I_{X_{C}}\right)=\lim _{m \rightarrow+\infty} \frac{\alpha\left(I_{X_{C}}^{(m)}\right)}{m}$ exists. Then from Theorem 3.9 we have

$$
\widehat{\alpha}\left(I_{X_{C}}\right)=\lim _{p \rightarrow+\infty} \frac{\alpha\left(I_{X_{C}}^{(p n)}\right)}{p n}=\lim _{p \rightarrow+\infty} \frac{p v}{p n}=\frac{v}{n} .
$$

Remark 3.13 The homological invariants do not depend on the choice of the hyperplanes, provided that we take them meeting properly. 


\section{Homological invariants of C-Steiner configurations of points: Hilbert function and graded Betti numbers}

In this section we describe the Hilbert function of a C-Steiner configuration of points. We recall some known definitions. For a finite set of points $X \subset \mathbb{P}^{n}$ the Hilbert function of $X$ is defined as the numerical function $H_{X}: \mathbb{N} \rightarrow \mathbb{N}$ such that

$$
H_{X}(i)=\operatorname{dim}_{k}\left(R / I_{X}\right)_{i}=\operatorname{dim}_{k} R_{i}-\operatorname{dim}_{k}\left(I_{X}\right)_{i}
$$

where $R=k\left[\mathbb{P}^{n}\right]$ and the first difference of the Hilbert function is defined by $\Delta H_{X}(i):=$ $H_{X}(i)-H_{X}(i-1)$. The $h$-vector of $X$ is denoted by

$$
h_{X}=h=\left(1, h_{1}, \ldots, h_{p}\right)
$$

where $h_{i}=\Delta H_{X}(i)$ and $p$ is the last index such that $\Delta H_{X}(i)>0$. If $I$ is the ideal of a set of points, analogously to the case of fat points, $\operatorname{reg}(I)$ denotes the Castelnuovo-Mumford regularity of $I$.

The next result gives us informations on the $h$-vector of a C-Steiner configuration of points $X_{C}$. Recall that given a Steiner system $(V, B)$ of type $S(t, n, v)$, the number of blocks is $|B|=\frac{\left(\begin{array}{l}v \\ t\end{array}\right)}{\left(\begin{array}{l}n \\ t\end{array}\right)}$ and that the last entry $h_{p}$ of $h_{X_{C}}$ is in degree $p=v-n$. With the previous notation, we have the following:

Proposition 4.1 If $(V, B)$ is a Steiner system $S(t, n, v)$, then the h-vector of $X_{C}$ is

$$
h_{X_{C}}=\left(1, n,\left(\begin{array}{l}
n+1 \\
n-1
\end{array}\right), \ldots,\left(\begin{array}{l}
v-2 \\
n-1
\end{array}\right),\left(\begin{array}{l}
v-1 \\
n-1
\end{array}\right)-|B|\right) .
$$

Proof From Theorem 3.9 we have $\alpha\left(I_{\Delta_{C}}\right)$ is $v-n$. From Remark 2.11, a set of minimal generators of degree $v-n$ has $|B|$ elements. So we only need to show that $h_{X_{C}}(v-n+1)=0$. This follows since $\sum_{j=0}^{v-n} h_{X_{C}}(j)=\left(\begin{array}{l}v \\ n\end{array}\right)-|B|=\operatorname{deg} X_{C}$.

The regularity of $I_{X_{C}}$ is an easy consequence of Proposition 4.1:

Corollary $4.2 \operatorname{reg}\left(I_{X_{C}}\right)=\alpha\left(I_{X_{C}}\right)+1=v-n+1$.

Let $(V, B)$ be a Steiner system of type $S(t, n, v)$. A minimal graded free resolution of $I_{X_{C}}$ will be written as

$$
\begin{aligned}
0 & \rightarrow \bigoplus_{j} R(-j)^{\beta_{n-1, j}\left(I_{X_{C}}\right)} \rightarrow \cdots \rightarrow \bigoplus_{j} R(-j)^{\beta_{1, j}\left(I_{X_{C}}\right)} \\
& \rightarrow \bigoplus_{j} R(-j)^{\beta_{0, j}\left(I_{X_{C}}\right)} \rightarrow R \rightarrow R / I_{X_{C}} \rightarrow 0
\end{aligned}
$$

where $R(-j)$ is the free $R$-module obtained by shifting the degrees of $R$ by $j$, i.e. so that $R(-j)_{a}=R_{a-j}$.

The number $\beta_{i, j}\left(I_{X_{C}}\right)$ is called the $(i, j)$-th graded Betti numbers of $I_{X_{C}}$ and equals the number of minimal generators of degree $j$ in the $i$-th syzygy module of $I_{X_{C}}$.

Another consequence of Proposition 4.1 is that

$$
\beta_{i, j}\left(I_{X_{C}}\right)=0 \text { for any } j-i>v-n+1 .
$$

This means that the nonzero graded Betti numbers only occur in two rows of the Betti table $\beta\left(I_{X_{C}}\right):=\left(\beta_{i, i+j}\left(I_{X_{C}}\right)\right)_{i, j}$ of $I_{X_{C}}$.

The next proposition excludes the existence of first syzygies in degree $v-n+1$. 
Proposition 4.3 If $(V, B)$ is a Steiner system of type $S(t, n, v)$, then

$$
\beta_{1, v-n+1}\left(X_{C}\right)=0 .
$$

Proof From Proposition 3.6 it is enough to show that $\beta_{1, v-n+1}\left(J_{C}\right)=0$. By contradiction, let $\alpha_{1}, \alpha_{2} \in B$ be two distinct blocks of the Steiner system $(V, B)$ such that the monomials $M_{1}:=M_{V \backslash \alpha_{1}}$ and $M_{2}:=M_{V \backslash \alpha_{2}}$ give a linear syzygy. Then $\operatorname{gcd}\left(M_{1}, M_{2}\right)$ has degree $v-n-1$. This implies $\left|\alpha_{1} \cap \alpha_{2}\right|=n-1 \geq t$, a contradiction to Definition 2.3 of Steiner system.

Proposition 4.4 If $(V, B)$ is a Steiner system of type $S(t, n, v)$, then

$$
\beta_{0, v-n+1}\left(I_{X_{C}}\right)=n|B|-\left(\begin{array}{c}
v \\
n-1
\end{array}\right)=n \frac{\left(\begin{array}{l}
v \\
t
\end{array}\right)}{\left(\begin{array}{c}
n \\
t
\end{array}\right)}-\left(\begin{array}{c}
v \\
n-1
\end{array}\right) .
$$

Proof It is matter of computation to show that

$$
\Delta h_{X_{C}}(v-n+1)=-\left(\begin{array}{c}
v-1 \\
n-1
\end{array}\right)+|B|
$$

and

$$
\Delta h_{X_{C}}(v-n)=\left(\begin{array}{l}
v-1 \\
n-1
\end{array}\right)-|B|-\left(\begin{array}{c}
v-2 \\
n-1
\end{array}\right)=\left(\begin{array}{l}
v-2 \\
n-2
\end{array}\right)-|B|
$$

and for $i<v-n$

$$
\Delta h_{X_{C}}(i)=\left(\begin{array}{c}
i+n-2 \\
n-2
\end{array}\right) \text {. }
$$

Then

$$
\Delta^{2} h_{X_{C}}(v-n+1)=-\left(\begin{array}{l}
v-2 \\
n-2
\end{array}\right)-\left(\begin{array}{l}
v-1 \\
n-1
\end{array}\right)+2|B|
$$

and

$$
\Delta^{2} h_{X_{C}}(v-n)=\left(\begin{array}{c}
v-3 \\
n-3
\end{array}\right)-|B|
$$

and for $i<v-n$

$$
\Delta^{2} h_{X_{C}}(i)=\left(\begin{array}{c}
i+n-3 \\
n-3
\end{array}\right)
$$

So we have

$$
\begin{aligned}
& \Delta^{n} h_{X_{C}}(v-n+1)=n|B|-\left[\left(\begin{array}{l}
v-1 \\
n-1
\end{array}\right)+\left(\begin{array}{l}
v-2 \\
n-2
\end{array}\right)+\cdots\left(\begin{array}{c}
v-n \\
0
\end{array}\right)\right]= \\
& =n \frac{\left(\begin{array}{l}
v \\
t
\end{array}\right)}{\left(\begin{array}{l}
n \\
t
\end{array}\right)}-\left(\begin{array}{c}
v \\
n-1
\end{array}\right) .
\end{aligned}
$$

Then the statement follows from Proposition 4.3.

Corollary 4.5 If $(V, B)$ is a Steiner system of type $S(t, n, v)$, then $\beta_{0, v-n+1}\left(I_{X_{C}}\right)=0$ if and only if $t=n-1$. 
Proof From Proposition 4.4 we have

$$
\begin{gathered}
\beta_{0, v-n+1}\left(I_{X_{C}}\right)=\Delta^{n} h_{X_{C}}(v-n+1)=n|B|-\left[\left(\begin{array}{c}
v-1 \\
n-1
\end{array}\right)+\left(\begin{array}{c}
v-2 \\
n-2
\end{array}\right)+\cdots\left(\begin{array}{c}
v-n \\
0
\end{array}\right)\right]= \\
=n \frac{\left(\begin{array}{l}
v \\
t
\end{array}\right)}{\left(\begin{array}{c}
n \\
t
\end{array}\right)}-\left(\begin{array}{c}
v \\
n-1
\end{array}\right) .
\end{gathered}
$$

If $t=n-1$ then we get

$$
\beta_{0, v-n+1}\left(I_{X_{C}}\right)=n \frac{\left(\begin{array}{c}
v \\
n-1
\end{array}\right)}{\left(\begin{array}{c}
n \\
n-1
\end{array}\right)}-\left(\begin{array}{c}
v \\
n-1
\end{array}\right)=0 .
$$

If $\beta_{0, v-n+1}\left(I_{X_{C}}\right)=0$ then we have

$$
\frac{(n-t) !}{(v-t) !}=\frac{1}{(v-n+1) !}
$$

and then

$$
v-n+1=\left(\begin{array}{l}
v-t \\
n-t
\end{array}\right)
$$

This implies

$$
\left(\begin{array}{c}
v-n+1 \\
v-n
\end{array}\right)=\left(\begin{array}{c}
v-t \\
v-n
\end{array}\right)
$$

and then $t=n-1$.

Corollary 4.6 If $t=n-1$, we have

$$
\beta_{i, j}\left(I_{X_{C}}\right)= \begin{cases}|B| & \text { if }(i, j)=(0, v-n) \\ (-1)^{i} \Delta^{n} h_{X_{C}}(v-n+1+i) & \text { if } j=v-n+1+i \\ 0 & \text { otherwise. }\end{cases}
$$

Corollary 4.7 We have

$$
\omega\left(I_{X_{C}}\right)= \begin{cases}\alpha\left(I_{X_{C}}\right)=v-n & \text { if } t=n-1 \\ \alpha\left(I_{X_{C}}\right)+1=\operatorname{reg}\left(I_{X_{C}}\right)=v-n+1 & \text { if } t<n-1\end{cases}
$$

where $\omega\left(I_{X_{C}}\right)$ is the largest degree in a minimal homogeneous set of generators of $I_{X_{C}}$.

Recalling that the resurgence and the asymptotic resurgence of $I$ are defined as $\rho(I):=$ $\sup \left\{\frac{m}{d} \mid I^{(m)} \nsubseteq I^{d}\right\}$ and $\rho_{a}(I)=\sup \left\{\frac{m}{r}: I^{(m t)} \nsubseteq I^{r t}\right.$ for all $\left.t>>0\right\}$, respectively, the following results give the bounds:

Corollary 4.8 Let $(V, B)$ be a Steiner system of type $S(t, n, v)$.

(1) If $t=n-1$ then

$$
\frac{(v-n) n}{v}=\rho_{a}\left(I_{X_{C}}\right) \leq \rho\left(I_{X_{C}}\right) \leq \frac{(v-n+1) n}{v} .
$$


(2) If $t<n-1$

$$
\frac{(v-n) n}{v} \leq \rho_{a}\left(I_{X_{C}}\right) \leq \rho\left(I_{X_{C}}\right) \leq \frac{(v-n+1) n}{v} .
$$

Proof By Theorem 1.2.1 in [4] and by Theorem 3.9 and Corollary 4.7 we have

$$
\frac{(v-n) n}{v}=\frac{\alpha\left(I_{X_{C}}\right)}{\widehat{\alpha}\left(I_{X_{C}}\right)} \leq \rho\left(I_{X_{C}}\right) \leq \frac{\operatorname{reg}\left(I_{X_{C}}\right)}{\widehat{\alpha}\left(I_{X_{C}}\right)}=\frac{(v-n+1) n}{v}
$$

Remark 4.9 From Lemma 2.3.4 in [4], if $d \operatorname{reg}\left(I_{X}\right) \leq \alpha\left(I_{X}^{(m)}\right)$, then $I_{X}^{(m)} \subseteq I^{d}$. Moreover, if $d \alpha\left(I_{X}\right)>\alpha\left(I_{X}^{(m)}\right)$ then $I_{X}^{(m)} \nsubseteq I_{X}^{d}$. The range of values not covered by these bounds has length

$$
\left\lfloor\frac{\alpha\left(I_{X}^{(m)}\right)}{\alpha\left(I_{X}\right)}-\frac{\alpha\left(I_{X}^{(m)}\right)}{\operatorname{reg}\left(I_{X}\right)}\right\rfloor=\left\lfloor\frac{\alpha\left(I_{X}^{(m)}\right)\left(\operatorname{reg}\left(I_{X}\right)-\alpha\left(I_{X}\right)\right)}{\alpha\left(I_{X}\right) \operatorname{reg}\left(I_{X}\right)}\right\rfloor
$$

For a C-Steiner configuration $X_{C}$, from Corollary 4.2, this number is equal to

$$
\left\lfloor\frac{\alpha\left(I_{X_{C}}^{(m)}\right)}{\alpha\left(I_{X_{C}}\right) \operatorname{reg}\left(I_{X_{C}}\right)}\right\rfloor .
$$

Example 4.10 We compute the $h$-vector and the graded Betti numbers of a C-Steiner System $X_{C}$ of type $S(2,3, v)$. We have $|B|=\frac{v(v-1)}{6}$ and then

$$
\begin{aligned}
& h_{X_{C}}=\left(1,3,6, \ldots,\left(\begin{array}{c}
v-2 \\
2
\end{array}\right),\left(\begin{array}{c}
v-1 \\
2
\end{array}\right)-|B|\right)= \\
& \Delta^{3} h_{X_{C}}=\left(1,0,0, \ldots, 0,-|B|, 0,2\left(\begin{array}{c}
v-1 \\
2
\end{array}\right)+(v-2)-3|B|,-\left(\begin{array}{c}
v-1 \\
2
\end{array}\right)+|B|\right)= \\
& =\left(1,0,0, \ldots, 0,-\frac{v(v-1)}{6}, 0, \frac{v(v-3)}{2},-\frac{(v-1)(v-3)}{3}\right) .
\end{aligned}
$$

In this case, we have: $\beta_{0, v-3}=\frac{v(v-1)}{6}, \beta_{1, v-2}=0, \beta_{1, v-1}=-\frac{v(v-3)}{2}$ and $\beta_{2, v}=$ $-\frac{(v-1)(v-3)}{6}$. In particular, $\alpha\left(I_{X_{C}}\right)=\omega\left(\left(I_{X_{C}}\right)=v-3\right.$ and $\operatorname{reg}\left(I_{X_{C}}\right)=v-2$.

In the following example we compute the graded Betti numbers of a C-Steiner configuration of type $S(t, n, v)$ where $t<n-1$.

Example 4.11 Consider a Steiner system $(V, B)$ of type $S(2,4,13)$ where $|V|=13$ and

$$
\begin{gathered}
B:=\{\{2,3,5,11\},\{3,4,6,12\},\{4,5,7,13\},\{1,5,6,8\},\{2,6,7,9\},\{3,7,8,10\}, \\
\{4,8,9,11\},\{5,9,10,12\},\{6,10,11,13\},\{1,7,11,12\},\{2,8,12,13\},
\end{gathered}
$$

$$
\{1,3,9,13\},\{1,2,4,10\}\} \text {. }
$$

Setting $C:=C_{(4,13)} \backslash V$, we construct a C-Steiner configuration $X_{C}$ in $\mathbb{P}^{4}$. We have

$$
h_{X_{C}}=(1,4,10,20,35,56,84,120,165,207) \text {. }
$$

We have $\beta_{0,9}=13, \beta_{0,10}=234, \beta_{1,11}=702, \beta_{2,12}=663, \beta_{3,13}=207$. In particular, we have $\alpha\left(I_{X_{C}}\right)=9$ and $\omega\left(I_{X_{C}}\right)=\operatorname{reg}\left(I_{X_{C}}\right)=10$.

In general, the following question is open. 
Question 4.12 Let $X_{C} \subseteq \mathbb{P}^{n}$ be a C-Steiner configuration of points of type $S(t, n, v)$. Do the graded Betti numbers and the Hilbert Function of $I_{X_{C}}^{(m)}$ only depend on the parameters $(t, n, v)$ ?

We don't have any formula to describe the Hilbert function of $I_{X_{C}}^{(m)}$. For $m=1$ Proposition 4.1 implies that the $h$-vector of $X_{C}$ is a pure $O$-sequence (see [39] for all the terminology and background on this topic). This is not always true if $m>1$. Using [1] and [32], we can show examples where the $h$-vectors could be not unimodal or not differentiable.

Example 4.13 Consider a Steiner system $\left(V_{1}, B_{1}\right)$ of type $S(2,3,7)$. Set $X_{C_{1}}:=X_{C_{(3,7)} \backslash B_{1}}$. Using [32], we found that $I_{X_{C_{1}}}^{(7)}$ has the following $h$-vector

$$
h_{I_{X_{C_{1}}}^{(7)}}=\left(1,3,6, \ldots, 153_{16}, 171, \mathbf{1 8 3}, \mathbf{1 8 2}, \mathbf{1 8 9}, 175,140,119,84,63,42,21,14_{28}\right)
$$

that is a non-unimodal sequence (the index denotes the degree in which the dimension occurs, for instance, above the entry 153 is in degree 16).

Example 4.14 Consider a Steiner configuration $\left(V_{2}, B_{2}\right)$ of type $S(2,3,9)$ and set $X_{C_{2}}:=$ $X_{C_{(3,7)} \backslash B_{2}}$. Using [32], we found that the $h$-vector of $I_{X_{C_{2}}}^{(10)}$ is

$$
\begin{aligned}
& h_{I_{X_{C_{2}}}^{(10)}}=\left(1,3,6, \ldots, 528_{31}, 561, \mathbf{5 8 3}, \mathbf{5 8 5}, \mathbf{6 0 3}, 621,639,621,567,540,540,528,468,396,\right. \\
& \left.360,360,324,252,216,216,204,144,108,108,108,72,36,36,36,24_{60}\right)
\end{aligned}
$$

that is unimodal but not differentiable (the positive part of the first difference is not an O-sequence).

We end this section with the following questions on $X_{\mathcal{H}, B}$ suggested by experimental evidences using [1] and [32]. From a combinatorial point of view, two Steiner systems having the same parameters could have very different properties. We have examples where such differences effect the homological invariants.

Question 4.15 Let $(V, B)$ be a Steiner system of type $S(t, n, v)$, and $X_{\mathcal{H}, B}$ the associated Steiner configuration of points. Assume that the hyperplanes in $\mathcal{H}$ are chosen generically. Do the Hilbert function and the graded Betti numbers of $X_{\mathcal{H}, B}$ only depend on $t, n, v$ ?

Question 4.16 Let $(V, B)$ be a Steiner system of type $S(t, n, v)$, and $X_{\mathcal{H}, B}$ the associated Steiner configuration of points. Assume that the hyperplanes in $\mathcal{H}$ are chosen generically. Are the Hilbert function and the graded Betti numbers of $X_{\mathcal{H}, B}$ generic with respect to the Hilbert function? (i.e. the same as a set of $|B|$ generic points in $\mathbb{P}^{n}$ ?)

\section{Application to coding theory}

In this section we show an application of the previous results on Steiner and C-Steiner configurations of points to coding theory. We recall the basic notion on linear coding and we compute the parameters of a linear code associated to a Steiner configuration and a C-Steiner configuration of points in $\mathbb{P}^{n}$. We refer to [15,24,52-55] for a detailed initial motivation to study the connections between the minimum distance and some invariants coming from commutative/homological algebra. There are several ways to compute the minimum distance. One of them comes from linear algebra. 
Let $k$ be any field and $X=\left\{P_{1}, \ldots, P_{r}\right\} \subseteq \mathbb{P}^{n}$ a not degenerate finite set of reduced points. The linear code associated to $\Gamma$ denoted by $\mathcal{C}(X)$ is the image of the injective linear $\operatorname{map} \varphi: k^{n+1} \rightarrow k^{r}$.

We are interested in three parameters $\left[|X|, k_{X}, d_{X}\right]$ that we use to evaluate the goodness of a linear code. The first number $|X|$ is the cardinality of $X$. The number $k_{X}$ is the dimension of the code as $k$-linear vector space, that is the rank of the matrix associated to $\varphi$. The number $d_{X}$ denotes the minimal distance of $\mathcal{C}(X)$, that is the minimum of the Hamming distance of two elements in $\mathcal{C}(X)$. The Singleton bound gives always an upper bound for this number: $d_{X} \leq|X|-n$. When $d_{X}=|X|-n$ the code is called a maximal distance separable code (MDS code).

The linear code associated to $X$ has generating matrix of type $(n+1) \times r$

$$
A(X)=\left[c_{1} \ldots c_{r}\right]
$$

where $c_{i}$ are the coordinates of $P_{i}$. Then the linear code $\mathcal{C}(X)$ has parameters $[|X|$, $\left.\operatorname{Rank}(A(X)), d_{X}\right]$. Assuming that $A(X)$ has no proportional columns is equivalent to say that the points $P_{i}$ are distinct points in $\mathbb{P}^{n}$. Then $|X|=r, \operatorname{Rank}(A(X))=n+1$ and $r-d_{X}$ is the maximum number of these points that fit in a hyperplane of $\mathbb{P}^{n}$. Remark 2.2 in $[52,53]$ says that the minimum distance $d_{X}$ is also the minimum number such that $r-d_{X}$ columns in $A(X)$ span an $n$-dimensional space. The generating matrix $A(X)$ of an $[|X|, n+1, d]$-linear code $\mathcal{C}$ naturally determines a matroid $M(\mathcal{C})$.

Denoted by $\operatorname{hy} p(X)$ the maximum number of points contained in some hyperplane, $d_{X}$ has also geometrical interpretation, that is $d_{X}=|X|-h y p(X)$, see Sect. 2 in [52] and Remark 2.7 in [54]. In particular, the authors borrowed this terminology from coding theory since $d_{X}$ is exactly the minimum distance of the (equivalence class of) linear codes with generating matrix having as columns the coordinates of the points of $X$.

Set $X_{B}:=X_{\mathcal{H}, B}$. We apply the results of the previous sections to compute the parameters of linear codes associated to both a Steiner configuration $X_{B}$ of points and its Complement $X_{C}$.

Proposition 5.1 Let $(V, B)$ be a Steiner system of type $S(t, n, v)$ with $|V|=v$. If $X_{B}$ is the Steiner configuration of points and $X_{C}$ its Complement, we have

$$
\operatorname{hyp}\left(X_{B}\right)=\frac{\left(\begin{array}{c}
v-1 \\
t-1
\end{array}\right)}{\left(\begin{array}{c}
n-1 \\
t-1
\end{array}\right)}
$$

and

$$
\operatorname{hyp}\left(X_{C}\right)=\left(\begin{array}{l}
v-1 \\
n-1
\end{array}\right)-\frac{\left(\begin{array}{c}
v-1 \\
t-1
\end{array}\right)}{\left(\begin{array}{c}
n-1 \\
t-1
\end{array}\right)}
$$

Proof Let $H_{1} \in \mathcal{H}$ be one of the hyperplanes involved in the construction of a Steiner configuration. Then it is clear by definition that the number of blocks in $B$ containing 1, i.e., the number of points in $H_{1}$ is

$$
\operatorname{hyp}\left(X_{B}\right)=\frac{\left(\begin{array}{c}
v-1 \\
t-1
\end{array}\right)}{\left(\begin{array}{c}
n-1 \\
t-1
\end{array}\right)}
$$


Since the star configuration $X_{\mathcal{H}, C_{(n, v)}}$ has $\left(\begin{array}{l}v-1 \\ n-1\end{array}\right)$ points lying on $H_{1}$, we get

$$
\operatorname{hyp}\left(X_{C}\right)=\left(\begin{array}{c}
v-1 \\
n-1
\end{array}\right)-\frac{\left(\begin{array}{c}
v-1 \\
t-1
\end{array}\right)}{\left(\begin{array}{c}
n-1 \\
t-1
\end{array}\right)} \text {. }
$$

Proposition 5.2 Let $(V, B)$ be a Steiner system of type $S(t, n, v)$ with $|V|=v$. Then a Steiner configuration of points $X_{B} \subseteq \mathbb{P}^{n}$ defines a linear code with

$$
d_{X_{B}}=\frac{\left(\begin{array}{l}
v \\
t
\end{array}\right)}{\left(\begin{array}{l}
n \\
t
\end{array}\right)}-\frac{\left(\begin{array}{c}
v-1 \\
t-1
\end{array}\right)}{\left(\begin{array}{l}
n-1 \\
t-1
\end{array}\right)} .
$$

A C-Steiner configuration of points $X_{C} \subseteq \mathbb{P}^{n}$ defines a linear code with

$$
d_{X_{C}}=\left(\begin{array}{l}
v \\
n
\end{array}\right)-\frac{\left(\begin{array}{l}
v \\
t
\end{array}\right)}{\left(\begin{array}{l}
n \\
t
\end{array}\right)}-\left(\begin{array}{l}
v-1 \\
n-1
\end{array}\right)+\frac{\left(\begin{array}{c}
v-1 \\
t-1
\end{array}\right)}{\left(\begin{array}{c}
n-1 \\
t-1
\end{array}\right)} .
$$

Proof The statement follows from Proposition 5.1 since, from Remark 2.7 in [54], for a set of points $X$ it is $d_{X}=|X|-h y p(X)$.

Remark 5.3 For a Steiner triple system $S(2,3, v)$ with block set $B$, define $C:=C_{(3, v)} \backslash B$. Then $X_{B}$ and $X_{C}$ have the following minimal distance

$$
d_{X_{B}}=\frac{\left(\begin{array}{l}
v \\
2
\end{array}\right)}{3}-\frac{v-1}{2}=\frac{(v-1)(v-3)}{6}
$$

and

$$
d_{X_{C}}=\left(\begin{array}{l}
v \\
3
\end{array}\right)-\frac{\left(\begin{array}{l}
v \\
2
\end{array}\right)}{3}-\left(\begin{array}{c}
v-1 \\
2
\end{array}\right)+\frac{v-1}{2}=\frac{(v-1)(v-3)^{2}}{6} .
$$

With the above results, we have

Theorem 5.4 Let $(V, B)$ be a Steiner system $S(t, n, v)$ with $|V|=v$. Then

(1) the parameters of the linear code defined by a Steiner configuration of points $X_{B}$ are $\left[|B|, n+1, d_{X_{B}}\right]$

(2) the parameters of the linear code defined by a Complement of a Steiner configuration of points $X_{C}$ are $\left[\left(\begin{array}{l}v \\ n\end{array}\right)-|B|, n+1, d_{X_{C}}\right]$.

Theorem 5.5 Let $(V, B)$ be a Steiner system $S(t, n, v)$ with $|V|=v$.

(1) If $n=\frac{\left(\begin{array}{c}v-1 \\ t-1\end{array}\right)}{\left(\begin{array}{c}n-1 \\ t-1\end{array}\right)}$ then $\mathcal{C}\left(X_{B}\right)$ is a MDS code;

(2) if $n=\left(\begin{array}{c}v-1 \\ t-1\end{array}\right)-\frac{\left(\begin{array}{c}v-1 \\ t-1\end{array}\right)}{\left(\begin{array}{c}n-1 \\ t-1\end{array}\right)}$ then $\mathcal{C}\left(X_{C}\right)$ is a MDS code;

Example 5.6 Consider the Steiner system $S(2,3,7)$. The blocks are, up to isomorphism, $B:=\{\{1,2,3\},\{1,4,5\},\{1,6,7\},\{2,4,6\},\{2,5,7\},\{3,4,7\},\{3,5,6\}\}$.

Let $\ell_{i}:=x+2^{i} y+3^{i} z+5^{i} w \in \mathbb{C}[x, y, z, w]=\mathbb{C}\left[\mathbb{P}^{3}\right]$ be linear forms and let $H_{i} \subseteq \mathbb{P}^{3}$ be the hyperplane defined by $\ell_{i}$ for $i=1, \ldots, 7$. Set $\mathcal{H}:=\left\{H_{1}, \ldots, H_{7}\right\}$. One can check that any three hyperplanes in $\mathcal{H}$ meet in one point. Computing with Cocoa [1] the generating matrix of the linear code $\mathcal{C}\left(X_{\mathcal{H}, B}\right)$ defined by the Steiner configuration on $B$ we get 


$$
A\left(X_{\mathcal{H}, B}\right):=\left(\begin{array}{ccccccc}
-15 & -1983 & -438045 & -350 & -639000 & 9315 & 104625 \\
20 & 1576 & 269060 & 160 & 240075 & -2610 & -25875 \\
-10 & -418 & -34230 & -35 & -37550 & 470 & 4250 \\
1 & 17 & 523 & 1 & 666 & -9 & -99
\end{array}\right)
$$

where the columns are the seven coordinates (among the thirty-five) of the intersection points of any three hyperplanes $H_{1}, \ldots, H_{7}$ corresponding to the seven blocks $B$. The parameters of the code $\mathcal{C}\left(X_{\mathcal{H}, B}\right)$ are $[7,4,4]$.

We note that only in this particular case, the linear code $\mathcal{C}\left(X_{\mathcal{H}, B}\right)$ associated to a Steiner system of type $S(2,3,7)$ has $d_{X_{B}}=4=7-3$ and $\mathcal{C}\left(X_{\mathcal{H}, B}\right)$ is a maximal distance separable code (MDS).

We now compute linear code $\mathcal{C}\left(X_{\mathcal{H}, C_{(3,7)} \backslash B}\right)$ associated to the Complement of the Steiner configuration. We get

$$
\begin{aligned}
A\left(X_{\left.\mathcal{H}, C_{(3,7) \backslash B}\right):=}\right. & \left(\begin{array}{ccccccccccccc}
75 & -207 & 615 & 4845 & -465 & -70 & 27585 & -190 & 21300 & -5985 & 36273 & -3610 \\
-90 & 232 & -660 & -5060 & 460 & 64 & -23980 & 160 & -16005 & 4340 & -24728 & 2368 \\
40 & -94 & 250 & 1830 & -170 & -21 & 7190 & -45 & 3755 & -930 & 4586 & -387 \\
-3 & 5 & -9 & -43 & 11 & 1 & -239 & 1 & -111 & 19 & -115 & 7 \\
& 225 & 1125 & -621 & 9225 & 6975 & 413775 & -29745 & -108819 & -3375 & -33750 & 5250 \\
& -150 & -675 & 348 & -4950 & -3450 & -179850 & 11820 & 37092 & 1125 & 10125 & -1200 \\
& 50 & 200 & -94 & 1250 & 850 & 35950 & -2090 & -4586 & -250 & -2000 & 175 \\
& -3 & -9 & 3 & -27 & -33 & -717 & 51 & 69 & 9 & 54 & -3 \\
& -446175 & -101250 & 1012500 & -3138750 & 3037500 \\
& 88650 & 16875 & -151875 & 388125 & -253125 \\
& -10450 & -2500 & 20000 & -42500 & 25000 \\
153 & 54 & -324 & 594 & -324
\end{array}\right)
\end{aligned}
$$

where the columns are the twenty-eight coordinates of the intersection points (among the thirty-five) of any three hyperplanes $H_{1}, \ldots, H_{7}$ corresponding to the twenty-eight blocks

$$
\begin{aligned}
C_{(3,7)} \backslash B= & \{\{1,2,4\},\{1,2,5\},\{1,2,6\},\{1,2,7\},\{1,3,4\},\{1,3,5\},\{1,3,6\},\{1,3,7\},\{1,4,6\}, \\
& \{1,4,7\},\{1,5,6\},\{1,5,7\},\{2,3,4\},\{2,3,5\},\{2,3,6\},\{2,3,7\},\{2,4,5\},\{2,4,7\}, \\
& \{2,5,6\},\{2,6,7\},\{3,4,5\},\{3,4,6\},\{3,5,7\},\{3,6,7\},\{4,5,6\},\{4,5,7\},\{4,6,7\},\{5,6,7\}\} .
\end{aligned}
$$

The parameters of the code $\mathcal{C}\left(X_{\mathcal{H}, C_{(3,7)} \backslash B}\right)$ are $[28,4,16]$.

Acknowledgements This project began in April 2018 when E. Guardo and L. Milazzo met together with the idea of making an interdisciplinary project between their own fields of research, such as Combinatorics and Commmutative Algebra/Algebraic Geometry. This is one of the two projects where L. Milazzo gave his contribution with the idea of involving other mathematicians, such as E. Ballico and G. Favacchio. There is another one, still not published and in progress, that involves other people from University of Catania. However, L. Milazzo became quite ill in February 2019, and after many years battle with his illness fought with huge strengh and courage, unexpectedly he passed away on March 4, 2019 in Catania. Lorenzo gave ideas to this paper, and turned his attention to this paper every time he could do it. Unfortunately, Lorenzo was not able to see the final version of this paper and we feel that his contributions warrant an authorship. Lorenzo's interests and contributions to the topics in this paper should be recognized. Lorenzo is greatly missed to all of us. The first author was partially supported by MIUR (Italy). G. Favacchio, E. Guardo and L. Milazzo's work has been supported by the Università degli Studi di Catania, "Piano della Ricerca 2016/2018 Linea di intervento 2". All the authors are members of GNSAGA of INdAM (Italy). We thank M. Gionfriddo, B. Harbourne, and S. Milici for their useful conversations on the topic and for their encouragement in finishing the project. We also thank Zs. Tuza for his useful comments in the revised version of the paper. The authors thank the two anonymous referees for their useful comments that improved the previous version of the paper.

Funding Open access funding provided by Università degli Studi di Catania within the CRUI-CARE Agreement.

Open Access This article is licensed under a Creative Commons Attribution 4.0 International License, which permits use, sharing, adaptation, distribution and reproduction in any medium or format, as long as you give 
appropriate credit to the original author(s) and the source, provide a link to the Creative Commons licence, and indicate if changes were made. The images or other third party material in this article are included in the article's Creative Commons licence, unless indicated otherwise in a credit line to the material. If material is not included in the article's Creative Commons licence and your intended use is not permitted by statutory regulation or exceeds the permitted use, you will need to obtain permission directly from the copyright holder. To view a copy of this licence, visit http://creativecommons.org/licenses/by/4.0/.

\section{References}

1. Abbott J., Bigatti A.M., Robbiano L.: CoCoA: a system for doing Computations in Commutative Algebra. Available at http://cocoa.dima.unige.it

2. Bocci C., Cooper S.M., Guardo E., Harbourne B., Janssen M., Nagel U., Seceleanu A., Van Tuyl A., Vu T.: The Waldschmidt constant for squarefree monomial ideals. J. Algebr. Comb. 44(4), 875-904 (2016).

3. Bocci C., Harbourne B.: The resurgence of ideals of points and the containment problem. Proc. Am. Math. Soc. 138(4), 1175-1190 (2010).

4. Bruns W., Herzog J.: Cohen-Macaulay Rings, vol. 39. Cambridge Studies in Advanced Mathematics, Revised Edition (1998).

5. Buratti M., Gionfriddo G., Milazzo L., Voloshin V.: Lower and upper chromatic numbers for $B S T S\left(2^{h}-\right.$ 1)s. Comput. Sci. J. Moldova 9(2), 259-272 (2001).

6. Buratti M., Rinaldi G., Traetta T.: 3-pyramidal Steiner triple systems. Ars Math. Contemp. vol 13, No. 1 (2017).

7. Carlini E., Catalisano M.V., Guardo E., Van Tuyl A.: Hadamard star configurations. Rocky Mount. J. Math 49(2), 419-432 (2001).

8. Carlini E., Guardo E., Van Tuyl A.: Star configurations on generic hypersurfaces. J. Algebra 407, 1-20 (2014).

9. Catalisano M.V., Guardo E., Shin Y.S.: The Waldschmidt constant of special $k$-configurations in $\mathbb{P}^{n}$. J. Pure Appl. Algebra. (2020). https://doi.org/10.1016/j.jpaa.2020.106341.

10. Chudnovsky G.V.: Singular points on complex hypersurfaces and multidimensional Schwarz Lemma. In: M-J Bertin (ed.) Seminaire de Theorie des Nombres, Paris 1979-80, Seminaire Delange-Pisot-Poitou, Progress in Math vol. 12. Birkhauser, Boston (1981).

11. Colbourn C.J., Dinitz J.H.: CRC Handbook of Combinatorial Designs. CRC Press, Boca Raton (2010).

12. Colbourn C.J., Pulleyblank W.R.: Matroid Steiner problems, the Tutte polynomial and network reliability. J. Comb. Theory Ser. B 47(1), 20-31 (1989).

13. Colbourn C.J., Rosa A.: Triple Systems. Oxford University Press, Oxford (1999).

14. Cooper S.M., Embree R.J., Há H.T., Hoefel A.H.: Symbolic powers of monomial ideals. Proc. Edinb Math. Soc. 60(1), 39-55 (2017).

15. Cooper S.M., Guardo E.: Fat points, partial intersections and Hamming distance. J. Algebra Appl. 19(4), 2050071 (2020). https://doi.org/10.1142/S0219498820500711.

16. Cooper S.M., Seceleanu A., Tohaneanu S., Vaz Pinto M., Villarreal R.H.: GMD functions for scheme based linear codes and algebraic invariants of Geramita ideals. Adv. Appl. Math. 112, 101940 (2020).

17. Czapliśki A., Glówka A., Malara G., Lampa-Baczyńska M., Luszcz-Swidecka P., Pokora P., Szpond J.: A counterexample to the containment $I^{(3)} \subseteq I^{2}$ over the reals. Adv. Geom. 16(1), 77-82 (2016).

18. Dao H., De Stefani A., Grifo E., Huneke C., Núñez- Betancourt L.: Symbolic powers of ideals. In: Singularities and Foliations. Geometry, Topology and Applications, pp. 387-432. Springer, Cham (2015).

19. De Boer M., Pellikaan R.: Groebner bases for codes. Some Tapas of Computer Algebra. Chap. 10, pp. 237-259. Springer, Berlin (1999).

20. Eisenbud D.: Commutative Algebra: With a View Toward Algebraic Geometry. Springer-Verlag, New York (1995).

21. Favacchio G., Guardo E., Migliore J.: On the arithmetically Cohen-Macaulay property for sets of points in multiprojective spaces. Proc. Am. Math. Soc. 146, 2811-2825 (2018). https://doi.org/10.1090/proc/ 13981.

22. Favacchio G., Guardo E., Picone B.: Special arrangements of lines: codimension two ACM varieties in $\mathbb{P}^{1} \times \mathbb{P}^{1} \times \mathbb{P}^{1}$. J. Algebra Appl. 18(4), 1 (2019).

23. Favacchio G., Migliore J.: Multiprojective spaces and the arithmetically Cohen-Macaulay property. Math. Proc. Cambr. Philos. Soc. 166(3), 583-597 (2019).

24. Garrousian M., Tohaneanu S.O.: Minimal distance of linear codes and $\alpha$-invariants. Adv. Appl. Math. 71, 190-207 (2015).

25. Geramita A., Harbourne B., Migliore J.: Star configurations in $\mathbb{P}^{n}$. J. Algebra 376, 279-299 (2013). 
26. Geramita A., Harbourne B., Migliore J., Nagel U.: Matroid configurations and symbolic powers of their ideals. Trans. Am. Math. Soc. 369(10), 7049-66 (2017).

27. Gionfriddo L.: A conjecture of Berge about linear hypergraphs and Steiner systems $S(2,4, v)$. Discret. Math. 255, 125-133 (2002).

28. Gionfriddo M., Milazzo L., Rosa A., Voloshin V.: Bicoloring Steiner systems $S(2,4, v)$. Discret. Math. 283, 249-253 (2004).

29. Gionfriddo M., Guardo E., Milazzo L.: Extending bicolorings for Steiner triple systems. Appl. Anal. Discret. Math. 7(2), 225-234 (2013).

30. Gionfriddo M., Guardo E., Milazzo L., Voloshin V.: Feasible sets of smalle bicolorable STSs. Austr. J. Math 59(1), 107-119 (2014).

31. Gold L., Little J., Schenck H.: Cayley-Bacharach and evaluation codes on complete intersections. J. Pure Appl. Algebra 196, 91-99 (2005).

32. Grayson D.R., Stillman M.E.: Macaulay 2, a software system for research in algebraic geometry

33. Guardo E., Harbourne B., Van Tuyl A.: Asymptotic resurgences for ideals of positive dimensional subschemes of projective space. Adv. Math. 246, 114-127 (2013). https://doi.org/10.1016/j.aim.2013.05. 027.

34. Hansen J.: Linkage and codes on complete intersections. Appl. Algebra Eng. Commun. Comput. 14(3), 175-185 (2003).

35. Herzog J., Hibi T.: Monomial Ideals. Springer, London (2011).

36. Lampa-Baczyńska M., Malara G.: On the containment hierarchy for simplicial ideals. J. Pure Appl. Algebra. 219(12), 402-412 (2015).

37. Lindner C.C., Rosa A.: Steiner quadruple systems-a survey. Discret. Math. 21, 147-181 (1978).

38. Martinez-Bernal J., Pitones Y., Villarreal R.H.: Minimum distance functions of complete intersections. J. Algebra Appl. (2017). https://doi.org/10.1142/S0219498818502043.

39. Migliore J., Nagel U., Zanello F.: Pure $O$-sequences: known results, applications, and open problems. In: Peeva I. (ed.) Commut. Algebra. Springer, New York (2013).

40. Milazzo L., Tuza Z.: Upper chromatic number of Steiner triple systems and quadruple systems. Discrete Math. 174, 247-259 (1997).

41. Milazzo L.: Strict colourings for classes of Steiner triple systems. Discret. Math. 182, 233-243 (1998).

42. Milazzo L., Tuza Zs: Logarithmic upper bound for the upper chromatic number of $S(t, t+1, v)$ systems. ARS Comb. 92, 213-223 (2009).

43. Milazzo L., Tuza Zs, Voloshin V.: Strict colorings of Steiner triple and quadruple systems: a survey. Discret. Math. 261, 399-411 (2003).

44. Milici S.: Colouring Steiner triple systems. Rendiconti del Seminario Matematico di Messina Serie II Tomo XXV Supplemento al n.8, 113-118 (2002).

45. Milici S., Rosa A., Voloshin V.: Steiner systems with specified block colour patterns. Discret. Math. 240, 145-160 (2001).

46. Minh N.C., Trung N.V.: Cohen-Macaulayness of monomial ideals and symbolic powers of Stanley-Reisner ideals. Adv. Math. 226(2), 1285-306 (2011).

47. Reid C., Rosa A.: Steiner systems S(2, 4,v)—a survey.

48. Smith G.G., Sturmfels B.: Combinatorial Algebraic Geometry: Selected Papers From 2016 Apprenticeship Program. Springer, Berlin (2017).

49. Swartz E.: Topological representations of matroids. J. Am. Math. Soc. 16(2), 427-42 (2003).

50. Szemberg T., Szpond J.: On the containment problem. Rend. Circolo Mat. Palermo. Ser. 66(2), 233-45 (2017).

51. Terai N., Trung N.V.: Cohen-Macaulayness of large powers of Stanley-Reisner ideals. Adv. Math. 229(2), 711-30 (2012).

52. Tohaneanu S.O.: The minimum distance of sets of points and the minimum socle degree. J. Pure Appl. Algebra. 215(11), 2645-51 (2011).

53. Tohaneanu S.O.: Lower bounds on minimal distance of evaluation codes. Appl. Algebra Eng. Commun. Comput. 20(5-6), 351 (2009).

54. Tohaneanu S.O., Van Tuyl A.: Bounding invariants of fat points using a coding theory construction. J. Pure Appl. Algebra. 217(2), 269-79 (2013).

55. Tohaneanu S.O., Xie Y.: Homological properties of ideals genertaed by $\alpha$-product of linear forms. (2019). https://128.84.21.199/pdf/1906.08346v3.pdf

56. van Lint J., van der Geer G.: Introduction to Coding Theory and Algebraic Geometry. Birkhäuser, Basel (2012).

57. Van Maldeghem H.: Some remarks on Steiner systems. Desig. Codes Cryptogr. 29, 199-213 (2003).

58. Varbaro M.: Symbolic powers and matroids. Proc. Am. Math. Soc. 139(7), 2357-66 (2011). 
59. Waldschmidt M.: Propriétés arithmétiques de fonctions de plusieurs variables II. Séminaire P. Lelong (Analyse), 1975/76, pp. 108-135. Lecture Notes Math., vol. 578. Springer, Heidelberg (1977)

Publisher's Note Springer Nature remains neutral with regard to jurisdictional claims in published maps and institutional affiliations. 